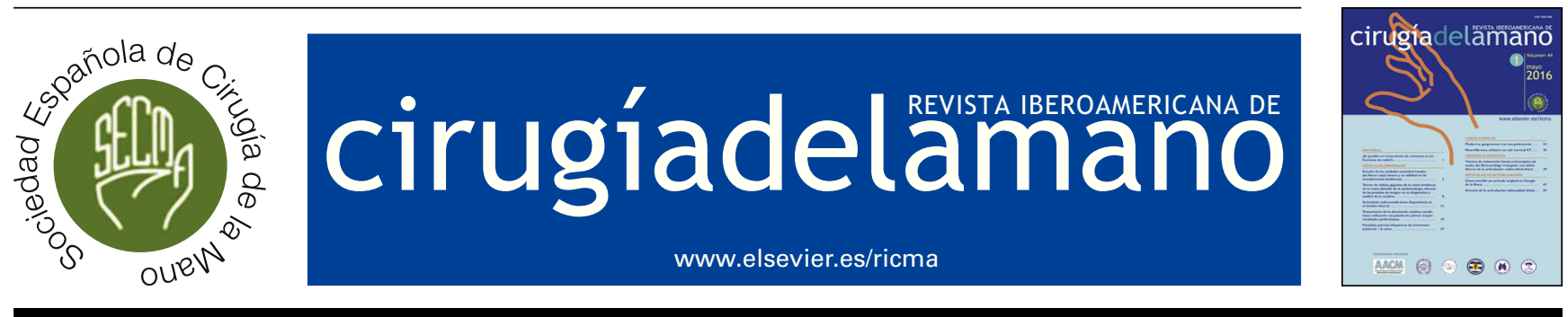

\title{
Fibroma sinovial como causa atípica de síndrome de túnel del carpo: presentación de 2 casos clínicos
}

\author{
X. Paredes-Carnero ${ }^{a, *}$, B. Gago Vidal ${ }^{\mathrm{b}}$ y P.J. Delgado ${ }^{\mathrm{c}}$ \\ a Servizo de Cirurxía Ortopédica e Traumatoloxía, Complexo Hospitalario Universitario de Ourense, Ourense, España \\ b Servizo de Cirurxía Plástica e Queimados, Hospital Povisa, Vigo, España \\ c Unidad de Cirugía de la Mano y Miembro Superior, Hospital Montepríncipe, Boadilla del Monte, Madrid, España
}

Recibido el 4 de mayo de 2016; aceptado el 30 de agosto de 2016

Disponible en Internet el 11 de octubre de 2016

\section{PALABRAS CLAVE \\ Síndrome del túnel del carpo; \\ Fibroma sinovial; Lesión ocupante de espacio}

\section{KEYWORDS}

Carpal tunnel syndrome;

Fibroma of the tendon sheath; Space occupying lesion

\begin{abstract}
Resumen El fibroma sinovial (FS) es una tumoración benigna que puede encontrarse en la mano, teniendo predilección por las vainas flexoras. Esta lesión puede comportarse como una lesión ocupante de espacio (LOE), la cual puede llegar a comprimir nervios, como en el túnel del carpo, provocando un síndrome del túnel del carpo (STC). Este hecho solo ha sido descrito en 9 ocasiones. Cuando el STC se presenta de manera unilateral debemos descartar una LOE, entre las cuales el FS debería ser considerado como diagnóstico diferencial, especialmente frente al tumor de células gigantes, del cual solo es diferenciable microscópicamente, por lo que la resección de la masa es imprescindible. Los 2 casos aquí descritos tuvieron diferentes presentaciones clínicas pero sin complicaciones tras la resección.

(C) 2016 SECMA. Publicado por Elsevier España, S.L.U. Este es un artículo Open Access bajo la licencia CC BY-NC-ND (http: / / creativecommons.org/licenses/by-nc-nd/4.0/).
\end{abstract}

Synovial fibroma as an atypical cause of carpal tunnel syndrome: A presentation of 2 clinical cases

Abstract The fibroma of tendon sheath (FTS) is a benign tumour that can be found in the hand, being more likely in the flexor sheaths. This can behave like a space occupying lesion (SOL), which can compress nerves, such as carpal tunnel, causing a carpal tunnel syndrome (CTS). This has only been described on 9 occasions. When CTS is presented unilaterally, a SOL must be ruled out, including the FTS in a differential diagnosis, especially against the giant cell tumour. This is only distinguishable microscopically, thus resection of the mass is essential. The 2 cases described here had different clinical presentations, but with no complications after resection. (C) 2016 SECMA. Published by Elsevier España, S.L.U. This is an open access article under the CC BY-NC-ND license (http://creativecommons.org/licenses/by-nc-nd/4.0/).

\footnotetext{
* Autor para correspondencia.

Correo electrónico: xavinef@gmail.com (X. Paredes-Carnero).
} 


\section{Introducción}

La etiología del síndrome del túnel del carpo (STC) incluye múltiples causas sistémicas (diabetes, hipotiroidismo, menopausia, embarazo, amiloidosis o artritis reumatoide) ${ }^{1}$, las cuales deben ser investigadas cuando se constate la bilateralidad del cuadro $^{2}$; sin embargo, en casos de afectación unilateral se deben descartar lesiones ocupantes de espacio (LOE). Nakamichi y Tachibana ${ }^{3}$ consideraron que hasta el $68 \%$ de los pacientes con alteraciones nerviosas unilaterales presentaban LOE como causa de STC, siendo las más frecuentes: lipomas, memangiomas, sarcomas sinoviales, gangliones, tuberculosis, calcificaciones o fibromas sinoviales (FS). Los FS son tumores benignos que se encuentran en el $98 \%$ de los casos en las extremidades, de los que el $81 \%$ lo hacen en el miembro superior y el $24 \%$ en la mano. El FS ha sido pocas veces descrito como causa de STC - tan solo 9 veces-, sin embargo es fundamental su cribado ante la sospecha de LOE para poder descartar así otras lesiones malignas.

A continuación se describen 2 casos clínicos de FS causantes de STC, que fueron diagnosticados en diferentes condiciones pero con tratamiento y resolución semejantes.

\section{Caso 1}

Varón de 80 años con antecedentes de HTA, hiperuricemia y FA crónica, en tratamiento con warfarina. Consultó en la Unidad de Mano de su hospital de referencia por un cuadro de pérdida de fuerza y parestesias en los dedos $1 .^{\circ}, 2 .^{\circ}$ y $3 .^{\circ}$ de la mano derecha de 4 años de evolución.

El principal síntoma del paciente era la pérdida de fuerza en los 3 primeros dedos que se producía desde hacía un año y medio, mientras que las parestesias comenzaron hacía 4 años, las cuales aumentaban por la noche.

En la exploración clínica se observó un signo de Tinel positivo en el territorio del túnel del carpo; hipotrofia de la musculatura tenar; masa de consistencia indurada en la cara volar inmediatamente proximal al pliegue palmar, de unos $3 \times 3 \mathrm{~cm}$, dolorosa a la palpación pero sin irradiación; engrosamiento de la fascia palmar de los dedos $3 .^{\circ}$ y $4 .^{\circ}$ de 2 y $3 \mathrm{~cm}$ respectivamente, condicionando la retracción en flexión de los correspondientes dedos, dando lugar a una enfermedad de Dupuytren grado II-III.

En cuanto a las pruebas complementarias, se observó en el EMG, una afectación severa $(25 \mathrm{~m} / \mathrm{s}$ de tipo motor y $15 \mathrm{~m} / \mathrm{s}$ de tipo sensitivo en el 3 er $^{\text {er }}$ radio) de tipo mixto del nervio mediano.

En el estudio ecográfico, en la región proximal del túnel del carpo, se objetivó una lesión de $2,3 \times 1,9 \times 1,3 \mathrm{~cm}$, de morfología ovalada, contorno bien definido, ecogenicidad heterogénea y sin flujo en el estudio Doppler. La lesión se encontraba en íntimo contacto con la vaina del tendón flexor común de los dedos, por lo que los diagnósticos de sospecha eran el tumor de células gigantes (TCG) de vaina tendinosa o el fibroma, aunque la posibilidad de un tumor neurogénico dependiente del nervio mediano tampoco se podía excluir.

Se llevó a cabo una intervención quirúrgica mediante un abordaje ampliado del túnel carpiano, desde $4 \mathrm{~cm}$ proximal al pliegue de la muñeca hasta la base de ambos dedos, realizando incisiones en $« Z$ » en los radios $3 .^{\circ}$ y $4 .^{\circ}$. Se extrajo

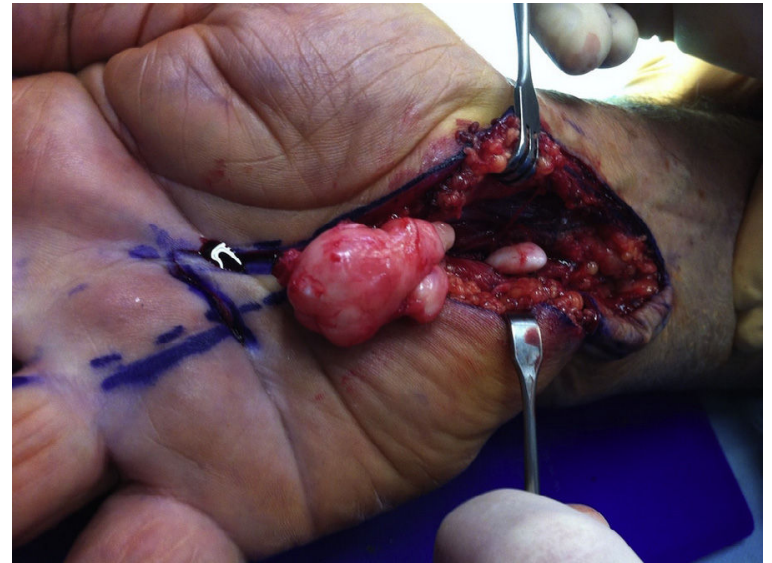

Figura 1 Imagen de la lesión del caso 1 una vez disecada la masa con el túnel del carpo abierto.

una masa de $2 \times 2 \mathrm{~cm}$ proximal al túnel del carpo que se extendía distalmente y se introducía en él. Se realizó un destechamiento del túnel y se observó la continuidad de la masa adelgazándose y prolongándose, adherida a la vaina del tendón flexor común profundo primero (fig. 1), y después a la del $3 .^{\text {er }}$ dedo hasta $3 \mathrm{~cm}$ proximal a la base de él. Se disecó dicha masa en toda su continuidad, siendo esta de aspecto lobulado y color blanquecino, con consistencia blanda. Tras su extracción se observó el nervio mediano, que a su paso por el túnel del carpo reducía su calibre y se volvía más blanquecino. Además se realizó la fasciectomía de los radios $3 .^{\circ}$ y $4 .^{\circ}$, disecando las cuerdas longitudinales y conservando las cuerdas transversales.

La anatomía patológica mostró abundantes células estrelladas y fusiformes en continuidad, lo que llevó a concluir que se trataba de un FS (fig. 2).

El paciente fue dado de alta el mismo día de la intervención, sin presentar complicaciones de la herida quirúrgica y aumentando progresivamente la movilidad de la mano. A los 2 meses de la intervención había recuperado parcialmente la fuerza en los dedos afectados, pero las parestesias nocturnas continuaron, aunque en menor cuantía. La movilidad completa de los dedos $3 .^{\circ}$ y $4 .^{\circ}$ se consiguió a las 3

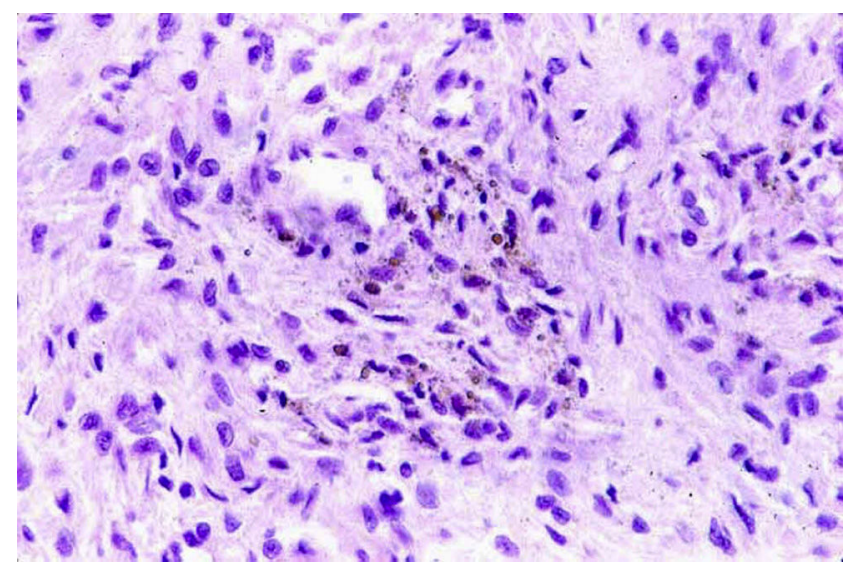

Figura 2 Imagen microscópica $(\mathrm{H}-\mathrm{E})$ de la muestra del caso 1 en donde podemos ver fibras de colágeno y fibroblastos rodeados de matriz mixoide, con pequeños vasos. 


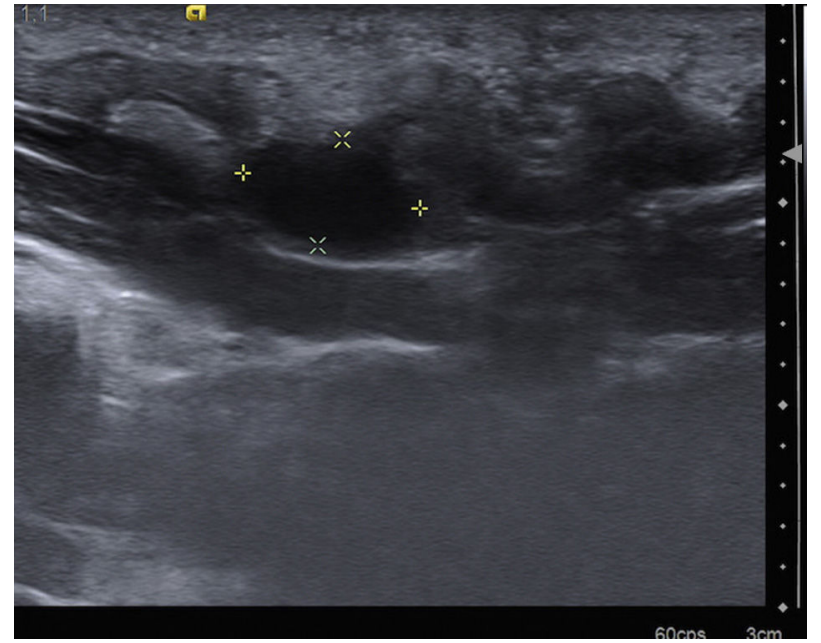

Figura 3 Ecografía del caso 2 en donde se puede observar una imagen nodular hipoecogénica en flexura de la muñeca de $0,8 \times 0,7 \times 0,7 \mathrm{~mm}$.

semanas. Pasados 6 meses desde la intervención, no ha presentado aumento de la clínica de STC, ni la aparición de una nueva masa, continuando con una movilidad completa en los dedos $3 .^{\circ}$ y $4 .^{\circ}$. Sin embargo la fuerza no se ha recuperado totalmente.

\section{Caso 2}

Varón diestro de 41 años sin antecedentes personales de interés. Su profesión es la de conductor de autobuses y como deportes ocasionales practica el pádel y el fitness. Consulta en la unidad de mano por parestesias en los dedos $2 .^{\circ}$ y $3 .^{\circ}$ de la mano derecha de 6 meses de evolución. Además se asociaban 2 masas de 2 meses de evolución: una en la muñeca y otra en la palma de la mano.

En la exploración clínica se observó un signo de Tinel y Phalen positivos. Existía una masa de consistencia indurada en la cara volar, inmediatamente proximal al pliegue palmar, de unos $1 \times 1 \mathrm{~cm}$, no dolorosa a la palpación, asociada a otra lesión en la cara volar de articulación MTC-F del $2 .^{\circ}$ dedo de consistencia indurada aunque menor que la anterior, de unos $0,5 \times 0,5 \mathrm{~cm}$.

En cuanto a las pruebas complementarias, se observó en el EMG una afectación sensitiva moderada $(35 \mathrm{~m} / \mathrm{s}$ en el 3 .er radio) del nervio mediano.

En el estudio ecográfico se observó una imagen nodular hipoecogénica en flexura de la muñeca de $0,8 \times 0,7 \times 0,7 \mathrm{~mm}$, subyacente al nervio mediano sin estar en continuidad con él (fig. 3). Asociada a la primera se observó otra imagen nodular hipoecogénica en el 2. ${ }^{\circ}$ espacio intermetacarpiano, de $0,6 \times 0,4 \times 0,5 \mathrm{~mm}$, en relación con la musculatura lumbrical e interósea.

Se llevó a cabo una intervención quirúrgica mediante un abordaje ampliado del túnel carpiano, que se extendía hasta el $2 .^{\circ}$ radio. Se extrajo una masa de $0,8 \times 0,7 \times 0,7 \mathrm{~cm}$ a unos $3 \mathrm{~cm}$ proximal al túnel del carpo (fig. 4). Se realizó un destechamiento del túnel y se observó la continuidad de la masa que se adelgazaba y prolongaba, adherida a la vaina del tendón flexor común profundo del $2 .{ }^{\circ}$ dedo, hasta $3 \mathrm{~cm}$ proximal

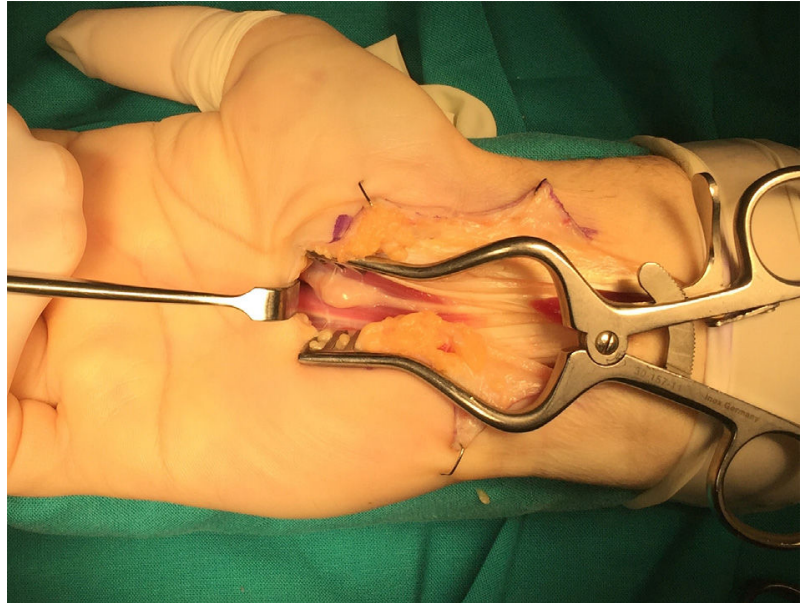

Figura 4 Vista intraoperaotoria del caso 2 en donde se puede observar la masa blanquecina adherida a la vaina de los flexores antes de ser extirpada.

a la base del dedo. Se extrajo una segunda masa a la altura de la articulación $\mathrm{MTC}-\mathrm{F}$ del $2 .^{\circ}$ radio de $0,6 \times 0,4 \times 0,5 \mathrm{~cm}$. Se disecó dicha masa en toda su continuidad, siendo esta de color blanquecino y consistencia pastosa (fig. 5). Tras su extracción se observó el nervio mediano, que a su paso por el túnel del carpo no presentaba cambios en su coloración o calibre.

La anatomía patológica mostró una lesión nodular con proliferación de células fusiformes de hábito fibroblástico en un estroma colagenizado sin atipia, compatible con FS.

El paciente fue dado de alta al día siguiente de la intervención. No presentó ninguna complicación postoperatoria y la movilidad de los dedos mejoró, hasta ser completa a los 10 días. Al día siguiente de la intervención las parestesias remitieron. El paciente permaneció con dolor en la

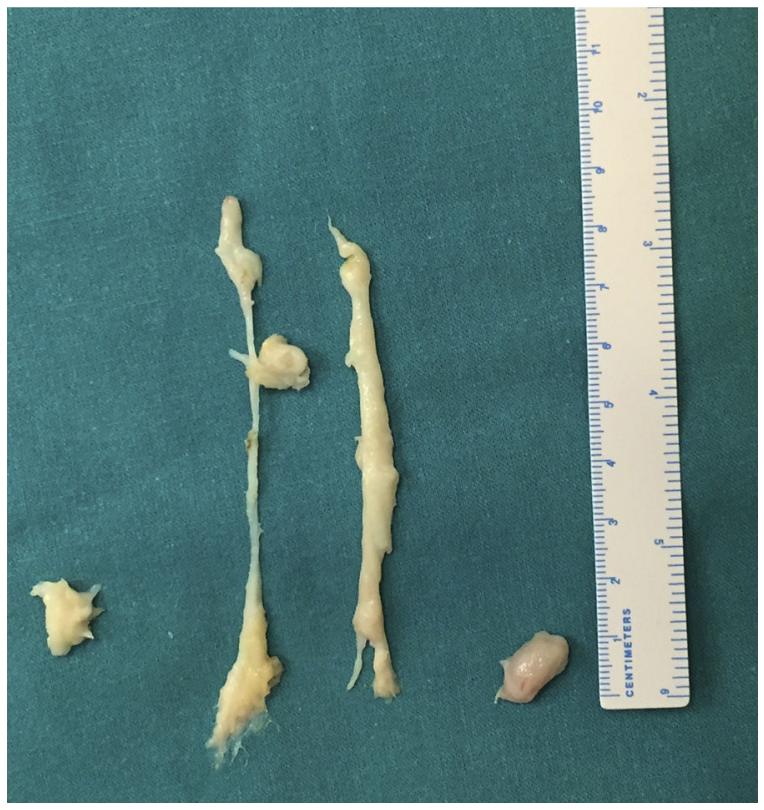

Figura 5 Muestra de las piezas extraídas del caso 2, en donde se observan las 2 masas extirpadas, así como la sinovitis del túnel, de aspecto semejante. 
eminencia tenar a la palpación hasta el mes de la intervención y con dolor leve en el antebrazo con la flexión de los dedos hasta los 2 meses de la intervención. El EMG a los 3 meses de la intervención no mostraba alteración en la

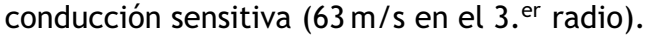

\section{Discusión}

El FS ha sido descrito en 9 ocasiones como causante de $\mathrm{STC}^{4-7}$. Se trata de un tumor benigno que fue descrito por Geschicker y Copelandin ${ }^{1}$ en 1936 por vez primera, pero no fue hasta 1979 cuando Chung y Enzinger ${ }^{8}$, tras revisar 138 casos, recogidos a lo largo de 48 años, concluyeron que su diagnóstico como TCG era erróneo y que se ajustaban a la descripción previa. Su localización preferente es la mano, al igual que los TCG, pero a diferencia de estos, los FS se sitúan en las vainas de los flexores, siendo el 1. ${ }^{\text {er }}$ dedo el de mayor prevalencia. En los casos aquí presentados los flexores afectados fueron los dedos $3 .^{\circ}$ en el primer caso y $2 .^{\circ}$ en el segundo.

Son tumores benignos de lento crecimiento, sin datos publicados de malignización. Afectan por igual a hombres y mujeres, aunque Chung y Enzinger los encontraron más frecuentemente en hombres comparados con los $\mathrm{TCG}^{8}$. Macroscópicamente son masas bien circunscritas, algunas veces encapsuladas y nodulares o multinodulares, que presentan un tamaño medio de $2 \mathrm{~cm}$. Sin embargo son difíciles de diferenciar de otras masas como el TCG, el ganglión, el neuroma, o incluso los sarcomas, sin realizar un análisis microscópico.

Es la observación microscópica la que dará el diagnóstico definitivo, siendo el principal diagnóstico diferencial con el TCG. A diferencia de este, los FS presentan densas e hipocelulares áreas de fibrocolágeno, con forma fusiforme y una matriz fibromixomatosa, con canales dilatados, con ausencia de células mitóticas y de necrosis.

Su resección permite la mejora de la sintomatología, pero la afectación severa del nervio mediano es un dato de mal pronóstico para la recuperación del paciente en los casos en que provoca un $\mathrm{STC}^{9}$. Su recurrencia se establece en torno al $24 \%$, entre el primer mes y el quinto año, produciéndose la mayoría de ellas entre el primer y el cuarto mes tras la resección ${ }^{8}$. Los 2 casos presentados no tuvieron recurrencias en el corto período de tiempo en el que fueron seguidos, 16 y 8 meses, respectivamente. Los 2 pacientes se comportaron de manera diferente en cuanto a la evolución de su STC. En el primer paciente, con años de evolución y una afectación severa del nervio mediano, tanto sensitiva como motora, la recuperación fue parcial, continuando con disestesias y sin recuperar la fuerza totalmente, mientras que el segundo paciente recuperó completamente la sensibilidad al tener una afectación leve del nervio mediano y presentar una clínica de corta duración. A pesar de la diferente evolución del STC, dependiente del momento del diagnóstico, no hubo complicaciones, confirmándose el carácter benigno de la lesión.
No existen casos publicados en los que se asocie este tumor con la enfermedad de Dupuytren, sin embargo, estudios no concluyentes sobre las causas del Dupuytren apuntan a factores estresantes como desencadenantes de la enfermedad, entre los que se encuentran traumatismos o compresiones, como sería este caso ${ }^{10}$.

Así pues, y como conclusiones, sería conveniente decir que el FS es un tumor benigno que afecta preferentemente a la mano, concretamente a las vainas flexoras. Su recurrencia es ocasional si se escinde completamente, sin ser frecuentes las complicaciones. Se debe establecer su diagnóstico diferencial fundamentalmente con el TCG, el cual solo puede ser realizado mediante el análisis microscópico tras la escisión de la masa. Si este tumor se sitúa en las vainas flexoras a nivel del túnel del carpo, puede causar un STC. Por eso, a pesar de ser esta una etiología poco frecuente de STC, debe ser tenida en cuenta en aquellos casos con clínica unilateral.

\section{Financiación}

En el presente artículo no ha habido fuente de financiación alguna, ya fuese pública o privada.

\section{Conflicto de intereses}

En el presente artículo no ha habido conflictos de interés entre los autores.

\section{Bibliografía}

1. Dailiana ZH, Bougioukli S, Varitimidis S, Kontogeorgakos V, Togia E, Vlychou M, et al. Tumors and tumor-like lesions mimicking carpal tunnel syndrome. Arch Orthop Trauma Surg. 2014;134:139-44.

2. Bagatur AE, Zorer G. The carpal tunnel syndrome is a bilateral disorder. J Bone Joint Surg Br. 2001;83:655-8.

3. Nakamichi K, Tachibana S. Unilateral carpal tunnel syndrome and space-occupying lesions. J Hand Surg Br. 1993;18: 748-9.

4. Evangelisti S, Reale VF. Fibroma of tendon sheath as a cause of carpal tunnel syndrome. J Hand Surg. 1992;17:1026-7.

5. Garrido A, Lam WL, Stanley PR. Fibroma of a tendon sheath at the wrist: A rare cause of compression of the median nerve. Scand J Plast Reconstr Surg Hand Surg. 2004;38:314-6.

6. Tiong WH, Ismael TS, Regan PJ. Fibroma of tendon sheath: A rare cause of carpal tunnel syndrome. J Hand Surg Br. 2006;31:579-80.

7. Rao J, Thoma A, Salama S. Fibroma of tendon sheath as a cause of carpal tunnel syndrome. Can J Plast Surg. 1997;5:176-8.

8. Chung EB, Enzinger FM. Fibroma of tendon sheath. Cancer. 1979;44:1945-54.

9. Kang HJ, Jung SH, Yoon HK, Hang SB, Kim SJ. Carpal tunnel syndrome caused by space occupying lesions. Yonsei Med J. 2009;50:257-61.

10. Green DP, Pederson WC, Hotchkiss RN, Wolfe SW. Green's operative hand surgery. 5th ed. New York: Elsevier; 2007. 